\title{
Chamber Wall Response to Target Implosion in Inertial Fusion Reactors: New and Critical Assessments
}

\author{
A. Hassanein and V. Morozov \\ Argonne National Laboratory \\ 9700 S. Cass Avenue-Bldg 308 \\ Argonne, IL 60439, USA
}

Tel: (630) 252-5889

Fax: (630) 252-3250

hassanein@anl gov

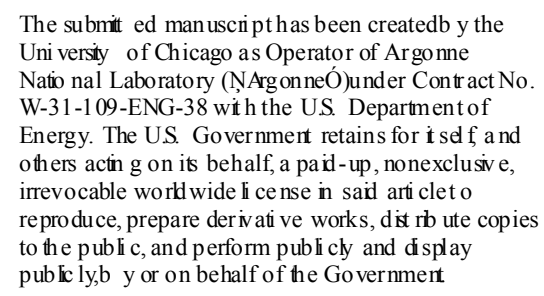

Presented at the Sixth International Symposium on Fusion Nuclear Technology (ISFNT-6), April 7-12, 2002, San Diego, CA USA 


\title{
Chamber Wall Response to Target Implosion in Inertial Fusion Reactors: New and Critical Assessments
}

\author{
A. HASSANEIN AND V. MOROZOV
}

Argonne National Laboratory, 9700 South Cass Avenue, Argonne, IL 60439, USA

\begin{abstract}
The chamber walls in inertial fusion energy (IFE) reactors are exposed to harsh conditions following each target implosion. Key issues of the cyclic IFE operation include intense photon and ion deposition, wall thermal and hydrodynamic evolution, wall erosion and fatigue lifetime, and chamber clearing and evacuation to ensure desirable conditions prior to target implosion. Several methods for wall protection have been proposed in the past, each having its own advantages and disadvantages. These methods include use of solid bare walls, gas-filled cavities, and liquid walls/jets. Detailed models have been developed for reflected laser light, emitted photons, and target debris deposition and interaction with chamber components and have been implemented in the comprehensive HEIGHTS software package. The hydrodynamic response of gasfilled cavities and photon radiation transport of the deposited energy has been calculated by means of new and advanced numerical techniques. Fragmentation models of liquid jets as a result of the deposited energy have also been developed, and the impact on chamber clearing dynamics has been evaluated. The focus of this study is to critically assess the reliability and the dynamic response of chamber walls in various proposed protection methods for IFE systems. Of particular concern is the effect on wall erosion lifetime of various erosion mechanisms, such as vaporization, chemical and physical sputtering, melt/liquid splashing and explosive erosion, and fragmentation of liquid walls.
\end{abstract}

\section{Introduction}

In inertial fusion systems, the power to the first wall resulting from energetic particles, neutrons, X-rays, and radiation is high enough to cause damage and dynamically affect the ability to reestablish chamber conditions prior to the next target implosion. In the case of a dry-wall protection scheme, the resulting target debris will interact and affect the surface wall materials in different ways. This situation could result in the emission of atomic (vaporization) and macroscopic particles (such as graphite or carbon composites), thereby limiting the lifetime of the wall. The mass loss in the form of macroscopic particles can be much larger than mass loss due to the surface vaporization and has not been properly considered in past studies as part of the overall cavity response and re-establishment. This could significantly alter cavity dynamics and power requirements.

The overall objective of this effort is to create a fully integrated model within the HEIGHTS software package to study chamber dynamic behavior after target implosion. This model includes cavity gas hydrodynamics; the particle/radiation interaction; the effects of various heat sources such as direct particle and debris deposition, gas conduction, convection, and radiation transport; chamber wall response and lifetime; and the cavity clearing. The model emphasizes the relatively long-time phenomena following 
the target implosion up to the chamber clearing in preparation for the next target injection. It takes into account both micro and macroscopic particles (mechanisms of generation, dynamics, vaporization, condensation, and deposition due to various heat sources: direct laser/particle beam, debris and target conduction, convection, and radiation). These processes are detrimental and of significant importance to the success of IFE reactors.

The experience gained from use of the HEIGHTS-MFE package which contains unique models and physics for magnetic fusion energy was applied to simulate the dynamics of chamber behavior in inertial fusion reactors. Various aspects of the HEIGHTS-MFE models have been benchmarked and tested against worldwide simulation devices and tokamak reactors in Japan, Europe, Russia, and the US. Besides magnetic fusion research, the HEIGHTS package has been used and is currently being applied to the space program (fire \& ice project), high-energy physics program (muon collider and neutrino factory projects), nuclear physics program (ATLAS project), and medical (isotope production and arc injury), and defense applications.

\section{Model considerations in an IFE reactor}

Following the micro-explosion in an IFE reactor, high energy x-rays and ions are produced and travel toward the chamber wall at high but differential speeds. Some of their energy is deposited in the residual or protective chamber gas, and is re-radiated to the wall over a longer time. Figure 1 shows a schematic of the energy deposition on the chamber wall. The main code models the transient hydrodynamics of the chamber gas, focusing on the relatively long-time phenomena following the target implosion up to the chamber clearing in preparation for the next target injection.

As a result of thermonuclear burn in inertially confined fusion (ICF) reactors, the first wall could be exposed to photon radiation and ion beam with a wide range of energies. The energy deposited in a material can be calculated from the mathematical relation for energy loss for each radiation type. Our study contains comprehensive analysis of these processes, including energy deposition from photons, ions, and laser beams.

The thermal response of the chamber wall exposed to thermonuclear radiation is determined when the time-and-space dependent energy depositions are known. Melting can occur in the case of a solid metallic during intense deposition of energy. Complexities in modeling this process arise as to the behavior of the melt layer under different loads and the resulting wall material loss. Mechanisms that contribute to melt layer loss are partially known and include effects such as melt splashing due to formation, boiling of volume bubbles that may result from continuous heating, and overheating of the liquid and other possible instabilities driven by various forces such as shock loading and gravity (1). Laboratory experiments on the effects of high heat fluxes and beam deposition on target materials have shown the formation of numerous liquid droplets that are splashed and lost during beam-target interaction.

Evaporation/sublimation increases substantially once the vaporization or sublimation temperature is reached. However, material vaporization also occurs at lower temperature and is dependent on the local conditions, such as surface temperature and the partial vapor pressure. The melt layer thickness is usually much larger than the surface 
vaporization. Therefore, splashing erosion from the developed melt layer could be quite important in determining the lifetime of IFE chamber walls.

Particle sputtering can be important in certain situations, depending on the impacting ion energy and chamber conditions. A physical module in the model has been developed to calculate chamber wall erosion due to particle debris bombardment. This physical sputtering can be an important erosion mechanism in ICF reactors.

Formation of volatile molecules on the target surface due to a chemical reaction between the incident particles and the target atoms is called chemical sputtering. It is especially observed for hydrogen and oxygen bombardment of graphite and carbon-based materials by the formation of hydrocarbon molecules, such as $\mathrm{CH}_{4}$ and $\mathrm{CO}$. In contrast to physical sputtering, chemical erosion strongly depends on the target temperature.

In carbon and carbon-based materials (CBMs), besides the enhanced temperaturedependent erosion yields by chemical sputtering around $800 \mathrm{~K}$, enhanced erosion yields were measured during ion bombardment at target temperatures above $1200 \mathrm{~K}$. This effect is known as radiation-enhanced sublimation. A model has been developed and implemented to calculate this effect as a function of wall temperature.

Macroscopic erosion may result from melt layer splashing of metallic components as well as from spallation (solid fragments) of brittle and carbon-based materials. In laboratory simulation experiments to study the effects of plasma instabilities in tokamak devices, macroscopic erosion dominated the erosion loss mechanism. Melt layer splashing occurred due to both melt layer superheating and hydrodynamic instabilities. Both mechanisms can exist during inertial fusion cavity response. Although macroscopic erosion is very complicated to model because many processes are involved, we are developing models that build on the experience gained from magnetic fusion as investigated earlier using the HEIGHTS package (2)

These processes must be taken into consideration to provide accurate net mass loss at each time step as input for the main hydrodynamic code. The actual condensation and redeposition rate of wall material will depend on the cavity conditions, as well as the type of erosion products. The interaction and redeposition of macroscopic erosion products are complicated and initial models are being developed to assess the geometrical effects of the cavity chamber on overall net wall erosion and on cavity clearance before the next target injection.

Currently proposed ICF reactor concepts have several design and operation features. Each concept employs a blast chamber in which the thermo-nuclear microexplosion occurs and is contained. Laser light or ion beams provide the heating and compression of the fuel target to ignition temperatures, and are directed into the blast chamber from final mirrors or focusing elements through ports located on the periphery of the cavity. As a result of the reaction, various fusion products are emitted and could impinge upon the blast chamber wall if the chamber is pumped to a hard vacuum. The thermonuclear burn of the fuel and the subsequent emission of fusion products, which strike the first wall, occur over a very short time (less than $10 \mathrm{~ns}$ ). As a result, a large amount of energy is deposited in the wall in very short time, and hydrodynamic stress waves are produced. One effect of the rapidly repeated micro-explosions is to quickly deteriorate any unprotected solid surfaces of the blast chamber. Therefore, some type of first-wall 
protection may be needed to maintain the structural integrity of the blast chamber. At the same time, the main objective of the ICF reactor is to efficiently convert heat, which is generated in the blast chamber and surrounding blanket, into usable energy. In addition to shielding the blast-chamber first wall, the protection system must permit rapid recovery of the energy in a form that is suitable for utilization in the energy conversion cycle. Thus, the first-wall protection system establishes many of the reactor design characteristics.

Most current reactor designs assume that the fuel target will contain a deuterium $(D)$ and tritium $(T)$ mixture, as well as some low $Z$ ablator (e.g., $C, O$ ) and high $Z$ (e.g., $F e$, $T a, P b$ ) elements. The fuel is compressed to the required conditions of temperature and density by the beam. The surface of the target is violently heated and ablated by intense beams. Very high pressure is generated, accelerating the fuel inward. The high $Z$ material carries the kinetic energy away from the microexplosion and moderates the alpha particles emitted as a product of the reaction. Ignition occurs when the rapidly moving inner region of the fuel is broken due to the pressure generated in the compressed matter, and the ignition temperature is reached.

The energy released is partitioned among different species: $\mathrm{x}$-rays, reflected laser light, alpha particles that have escaped the plasma, plasma debris, and neutrons. The plasma debris consists of both fast and debris ion fluxes. The energy of this debris is nearly a Maxwellian distribution with an average energy equal to the energy deposited in the target from the laser or ion beam plus the fraction of the thermonuclear reaction energy divided by the number of target particles. Also, in case of a laser driven-system, the laser light will contribute to the total energy released through a reflection mechanism. The energy spectrum for the x-rays may vary over a wide range. Thus, the energy partition and energy spectra depend upon the target design. Energy deposition from xray and fast and debris particles occurs near surfaces of incidence in structural and coolant materials, whereas the energy of neutrons is deposited through relatively large material volumes. The interior surface of the cavity wall would have to withstand repeated high yield energy deposition, and a very high surface temperature increase would result. Tolerable surface-temperature increases of such structural components have not been established either theoretically or experimentally.

A major goal of our analysis is to evaluate the fine details of the near surface evolution of the chamber wall, which includes temperature rise, erosion rate, physical and chemical sputtering, radiation-enhanced sublimation, evaporation, and melting. The temperature wall distribution is computed as a function in space mesh points. Therefore, the spatial mesh distribution must be treated very carefully. A uniform mesh is known to be sufficiently accurate for most applications, providing stable and fast calculation. At the same time, our physical problem requires that particular attention should be paid to calculation near the surface, particularly when the composite wall changes its properties.

\section{Photon interaction and deposition}

\subsection{Background}

The first wall can encounter photon radiation levels that range from a few eV to a few $\mathrm{MeV}$. The primary interaction of photons with materials in these energy ranges includes 
the photoelectric effect, coherent and incoherent scattering, and pair production. Cross sections for each of these reactions have been tabulated in various forms and are available for numerical calculations.

The HEIGHTS-IFE package calculates the volumetric energy deposition for a given $\mathrm{x}-$ ray spectrum or monoenergetic photons. Photon spectra may be specified as blackbodies or in histogram form. Deposition is based on general photoelectric and incoherent cross section libraries that have been incorporated into the package. The wall temperature response can be calculated for an adiabatic case, an impulse solution, and a finite duration deposition.

The wall response to photon deposition can be determined if the photon spectrum is specified. However, this spectrum depends on the target design and can only be described by very sophisticated and, therefore, quite expensive methods of calculation. In reactor design studies, the response of the wall can also be determined if the photons are characterized by common spectral forms or fitting functions. An example of photon spectra for a Naval Research Laboratory (NRL) target output is shown in Figure 2, where the photon spectrum information can be represented in the form of a threetemperature fitting function. The figure also compares photon spectra from indirect target output and $1 \mathrm{keV}$ blackbody spectrum.

Under certain conditions, the wall loading from source photons will occur at a time equal to the cavity radius divided by the speed of light. This statement is only true for a medium where the dielectric constant is independent of the frequency, so that the propagation of all energies will be at the same velocity. The deposition time for the x-ray energy spectrum is assumed to be between 1 and $10 \mathrm{~ns}$.

The deposition of x-rays into first wall materials will strongly depend on the energy spectrum of these x-rays. Soft x-rays deposit their energy within a micrometer of the wall's surface, very rapidly heating a thin layer of the first wall to a higher temperature. Harder x-ray energy spectra penetrate relatively longer distances into material, therefore heating a larger mass to a lower temperature.

\subsection{Calculations}

In the HEIGHTS numerical simulation, the deposition function and the total x-ray yield that is deposited on the wall are calculated by several methods using various space meshes. The purpose is to check the influence of mesh size on the amount of x-ray absorption.

Numerical simulation results of target implosion were obtained by means of ARIES spectra information for an NRL direct drive target (total yield 2.14 $\mathrm{MJ}$ ) deposited in both a carbon fiber composite (CFC) and tungsten wall. As shown in Figure 3 the CFC allows X-rays to penetrate more deeply through. As a result, a lower temperature rise is expected.

The HEIGHTS-IFE package can also represent the chamber wall in a multicomponent structure. For example, Figure 4 shows the absorption of NRL photon spectra in a composite $\mathrm{Li} / \mathrm{Pb}$ film on carbon structure. 


\section{Ion interaction and deposition}

\subsection{Background}

Ion deposition was calculated by means of several comprehensive models to predict the behavior and the slowing down of the incident ion flux in various candidate wall materials. In addition, sufficiently reasonable approximations were made by using Maxwellian or Gaussian distributions as well as a histogram. Tabulated results were easily accommodated in HEIGHTS in the form of histogram input to these calculations.

For some studies, target computer simulation predicts output spectra in the form of tabulated outcome. The NRL target simulation code supplies ion spectrum information in the form of a histogram. The histogram spectrum shows the distribution of incoming flux depending on the energy of particles.

Typical ion spectra from target output implosion calculations are shown in Figure 5 . These spectra are the result of NRL simulation of a thermonuclear target implosion within a total yield of $154 \mathrm{MJ}$.

The interaction of light ion beams $\left(\mathrm{H}, \mathrm{D}, \mathrm{T}, \mathrm{He}^{3}, \mathrm{He}^{4}\right)$ with the wall has been carefully studied by many authors because of its significance for fission and high-energy physics applications. The interaction of charged particles with materials primarily involves two processes. The first interaction is between the incident ion and the electrons in the wall material, which is an inelastic collision. The second interaction is between the collisions of the ions with material nuclei, which is an elastic interaction. The dominant mechanism of ions slowing down in materials depends upon the instantaneous energy of the moving ion. Several extensive methods are used in our calculations of the deposition and interaction of fast and debris ions with wall materials. These methods have been compared, and the range of validity of each method is well established (21)

\subsection{Calculations}

Below are the results obtained from HEIGHTS-IFE numerical calculations of the stopping power for the direct NRL target spectra from two wall materials, carbon and tungsten. Figure 6 shows the loss of fast and debris energy in the tungsten wall, while Figure 7 shows the same but for the graphite wall. HEIGHTS-IFE was also able to simulate ion deposition in composite wall structures. Figure 8, for example, presents the numerical simulation for a lithium/CFC wall bombarded by both the fast and debris ions. The ion spectra are also those taken from the NRL direct drive target. Particular attention must be taken when computing the total ion deposition function since each separate ion species arrives and deposits its energy at different times.

\section{Thermal evolution of the chamber wall}

The thermal response of a wall material exposed to thermonuclear radiation can be determined when all the time-and space-dependent energy-deposition functions are known. The aim of this section is to describe the numerical methods used in HEIGHTS for solving the heat diffusion equation subject to several moving and boundary conditions. All thermal properties of the composite wall structures are assumed to vary with temperature. 
The rapid heating of first-wall components due to x-ray and ion debris deposition in ICF reactors may lead to melting and subsequently to surface evaporation. As a result, an accurate analysis of this heat conduction problem initially requires the solution of at least two moving boundary problems. A moving front where vaporization occurs becomes one boundary, in addition to the moving internal boundary between the liquid and solid. Because of the moving boundary and the difference between the properties of the liquid and solid states of the same material, the distribution is nonlinear.

HEIGHTS-IFE calculates the wall thermal evolution in fine detail. The time evolution starts from the arrival of the x-rays, then the reflected laser lights, then the neutrons, then the fast and slow ion debris. Also, in the case of a gas-filled cavity, the re-radiated absorbed gas energy can be taken into account as a surface heat flux.

The surface temperature is determined by both the boundary conditions and the kinetics of the evaporation process. The correct boundary conditions entail partitioning of the incident energy flux into conduction, melting, evaporation, and possible radiation flux. The kinetics of evaporation establishes the connection between the surface temperature and the net atom flux leaving the surface taking into account the possibility of condensation flux.

Calculated results for the surface temperature of carbon and tungsten wall materials are presented in Figure 9. This calculation is for the bare-wall concept with no protection and for the lower yield NRL direct target spectra shown in Figure 5. Figure 9 also shows the time evolution of the wall thermal response due to the sequence of different incident species. The 3-D distribution of the surface temperature in both time and depth is shown in Figure 10.

\section{Erosion processes}

The erosion mechanisms of debris/surface interaction include physical sputtering, chemical sputtering, and radiation-enhanced sublimation. High-Z materials, such as tungsten, show low effective sputtering yield at low ion energies and, therefore, may be the preferred choice. For higher ion energies and low- $Z$ materials, such as lithium- or carbon-based materials, the sputtering is less critical, but chemical erosion may become important and cause additional wall erosion.

\subsection{Physical sputtering}

Physical sputtering is the removal of surface atoms from a solid due to the impact of energetic particles. The sputtering process can be described by momentum transport in a collision cascade initiated by the incident particle in the surface layer of the solid. A surface atom is ejected if the cascade of atoms reaches the surface with energy larger than the surface binding energy [9-13].

Physical sputtering is measured by the sputtering yield $Y$, defined as the mean number of atoms removed from the surface layer of the wall per incident ion. The energy of incident ions must be larger than a threshold energy for sputtering to occur, which is determined by the surface binding energy of the atoms of the wall and the momentum transfer process. Sputtering yields and their dependence of the incident ion energy, mass, and angle have been investigated experimentally by many authors. Computer 
simulation programs, such as the ITMC Monte Carlo code (part of the HEIGHTS package), can be used for more detailed numerical calculations.

Relatively high incident particle energies in the ICF condition will likely cause lower sputtering yield. However, if a gas is employed for cavity protection without sufficient density to stop these energetic ions, it may result in higher sputtering erosion. The HEIGHTS-IFE package is able to study that in detail (21).

\subsection{Chemical sputtering}

Chemical sputtering involves the formation of volatile molecules on the target surface due to a chemical reaction between the incident particles and the target atoms. It is especially observed for hydrogen and oxygen bombardment of graphite and carbon-based materials by the formation of hydrocarbon molecules, such as $\mathrm{CH}_{4}$ and $\mathrm{CO}$. For oxygen irradiation of carbon, erosion yields close to unity are found nearly independent on the incident energy and target temperature. For hydrogen irradiation of carbon, the chemical sputtering significantly depends on the target temperature and the incident energy. As shown by (18), chemical erosion yield reaches its maximum around $800 \mathrm{~K}$. At low ion energies (100 eV and less) the maximum decreases, and the temperature dependence becomes broader such that at room temperature the chemical sputtering yield may exceed the values of physical sputtering.

\subsection{Radiation- enhanced sublimation}

Physical sputtering is generally independent of the target temperature. For graphite, however, besides the enhanced erosion yields by chemical sputtering around $800 \mathrm{~K}$, enhanced erosion yields were measured for any ion bombardment at target temperatures above $1200 \mathrm{~K}$. This effect, named radiation-enhanced sublimation (RES), has been found mainly for carbon and carbon-based materials. This process results when interstitial atoms formed by the incident particles diffuse to the surface and sublimate.

\subsection{Erosion numerical results}

Figure 11 presents results of HEIGHTS-IFE numerical simulation of various erosion mechanisms for the NRL direct target case. Shown separately are the erosion rates caused by both fast proton $\left(0.54 M J \mathrm{H}_{\text {fast }}\right.$ yield $)$ and debris proton $\left(0.11 M J \mathrm{H}_{\text {debris }}\right.$ yield) is shown separately in Figure 11 As can be deduced from the figure for graphite, physical erosion is at least two orders of magnitude lower than chemical erosion and RES. Figure 12 compares the chemical, radiation-enhanced sublimation, and physical sputtering to incident particle flux of carbon and tungsten.

\section{Macroscopic erosion and brittle destruction}

Modeling predictions indicate that surface vaporization losses of metallic materials can be lowered by different protection schemes. However, for liquid metal surfaces, ablation is predicted theoretically to be in the form of macroscopic metal droplets due to splashing of the molten layer.

Laboratory simulation experiments to predict erosion of candidate wall materials during a plasma disruption in magnetic fusion systems have also shown that erosion of 
metallic materials (such as $\mathrm{W}, \mathrm{Be}, \mathrm{Al}$, and $\mathrm{Cu}$ ) can be much higher than mass losses due only to surface vaporization. The mass losses are in the form of liquid metal droplets with average sizes of few tens of micrometers leaving the target surface with velocities $\mathrm{V} \approx$ $10-50 \mathrm{~m} / \mathrm{s}$. Such ablation occurs as a result of splashing of the liquid layer mainly caused by boiling and explosion of gas bubbles in the liquid, absorption of debris momentum, and hydrodynamic instabilities (such as Kelvin-Helmholtz and Rayleigh-Taylor instabilities) developed in the liquid layer from various forces. Volume bubble boiling usually occurs from overheating of the liquid metal above the vaporization temperature at which saturation pressure is equal to the outer pressure of the vapor plasma above the exposed target surface.

Nonmetallic materials such as graphite and CBMs have also shown large erosion losses, significantly exceeding that from surface vaporization. This has been observed in different laboratory simulation facilities such as electron beams, lasers, plasma guns, and other devices. This macroscopic erosion of CBMs depends on several main parameters, such as net power flux to the surface, exposure time, and threshold energy required for brittle destruction. Therefore, more relevant experimental data and additional detailed modeling are needed to evaluate the erosion of $\mathrm{CBMs}$, which strongly depends on the type of carbon material.

The ejected macroscopic particles from CBMs or splashed droplets from liquid surfaces will also form an aerosol cloud near the target surface. Therefore, accurate calculations of mass losses require full description of the media near the wall surface, which consist of a mixture of vapor and droplets/macroscopic particles moving away from the surface.

\section{Conclusions}

Models have been developed to study the dynamic behavior of ICF cavities following target implosions. These models take into account energy deposition from the reflected laser light, emitted photons, neutrons, and target ion debris and the interaction/thermal evolution of chamber gas/wall components. The models are implemented in the comprehensive HEIGHTS-IFE package. The hydrodynamic response of gas-filled cavities and photon radiation transport of the deposited energy can also be accurately calculated by new and advanced numerical techniques. Fragmentation models of thick or thin liquid jets as a result of the deposited energy have also been developed, and the impact on chamber clearing dynamics has been evaluated using HEIGHTS-IFE.

The focus of this study was to critically assess erosion-causing mechanisms. Of particular concern was the effect on wall erosion lifetime of vaporization, chemical and physical sputtering, melt/liquid splashing, and macroscopic erosion. Depending on target yield and cavity gas pressure, most of these erosion mechanisms could be important factors in determining the overall lifetime of chamber walls.

\section{Acknowledgments}

This work is supported by the U.S. Department of Energy, Office of Fusion Energy Sciences. 


\section{References}

(1) A. Hassanein, Stability and Erosion of the Melt Layer Formed during Plasma Disruption, Fus. Technol. 15 (1989) 513.

(2) A. Hassanein and I. Konkashbaev, Comprehensive physical models and simulation package for plasma/material interactions during plasma instabilities, J. Nucl. Mater. 273 (1999) 326.

(3) W.G. Wolfer and A.M. Hassanein, On Melt Layer Stability Following a Plasma Disruption, J. Nucl. Mat. 111-112 (1982) 560-565.

(4) A. M. Hassanein, G.L. Kulcinski, and W.G. Wolfer, Surface Melting and Evaporation during Disruptions in Magnetic Fusion Reactors, Nuclear Engineering and Design/ Fusion 1 (1984) 307-324.

(5) A. M. Hassanein, G.L. Kulcinski, and W.G. Wolfer, Dynamics of Melting, Evaporation, and Resolidification of Materials Exposed to Plasma Disruptions, J. Nucl. Mater. 111-112 (1982) 554-559.

(6) A. Hassanein and I. Konkashbaev, Hydrodynamic Effects of Eroded Materials of Plasma-Facing Component during a Tokamak Disruption, J. Nucl. Mater. 283-287 (2000) 1171-1176.

(7) A. Hassanein et al., Erosion of Melt Layers Developed During a Plasma Disruption, Proceedings of the 18th Symposium on Fusion Technology, Vol. 1 (1994) 223-226.

(8) A. Hassanein, Simulation of Plasma Disruption Induced Melting and Vaporization by Ion or Electron Beam, J. Nucl. Mater. 122-123 (1984) 1453-1458.

(9) A. Hassanein, Erosion and Redeposition of Divertor and Wall Materials during Abnormal Events, Fus. Technol. 19 (1991) 1789.

(10) P. Sigmund. Phys. Rev., 184 (1969) 383.

(11) W. Eckstein, J. Bohdansky, and J. Roth, in Atomic and Plasma-Material Interaction Data for Fusion, Suppl. Nucl. Fusion 1 (1991) 51.

(12) J. Roth, Sputtering of Solids at Ion Bombardment, NATO-ASI Series B, Phys., eds D.E. Post and R. Behrisch, Plenum, New York, 131 (1986) 351.

(13) R. Behrish, ed., Sputtering by Particle Bombardment I, Topics in Applied Physics, Springer, Berlin, p. 47 (1981).

(14) J. Bohdansky, Nucl. Instr. and Meth. 2 (1984) 587. 
(15) J.P. Biersak and W. Eckstein, Appl. Phys., 34 (1984) 73.

(16) D.L. Smith, Physical Sputtering Model for Fusion Reactor First-Wall Materials, Trans. A, Nucl. Soc.27 (1977) 265.

(17) J. Bohdansky, Proc. SOFT Conference, Utrech (1988)

(18) C. García-Rosales, Erosion Processes in Plasma-Wall Interactions, J. Nucl. Mater., 211 (1994) 204.

(19) J. Roth, E. Vietzke, and A.A. Haasz, in Atomic and Plasma-Material Interaction Data for Fusion, Suppl. Nucl. Fusion 1 (1991) 63

(20) J. Bohdansky and J. Roth, Fusion Technology, eds., A.M. Van Ingen, A. Nijsen-Vis and H.T. Kippel, Elsevier, Amsterdam p. 889 (1989).

(21) A. Hassanein and V. Morozov, Development of Comprehensive and Integrated Model for IFE Cavity Dynamics, Argonne National Laboratory, Report ANLET/02/04, February 2002. 


\section{Figure captions}

Figure 1. Schrematic of energy deposition on chamber walt.

Figure 2. Photon speetra from direet and indireet target output and blackbody spectrum.

Figure 3. X-ray deposition in graphite and tungsten walls.

Figure 4. X-ray deposition incomposite wall strueture.

Figure 5. Particte energy distribution for ARL direct dirive target of 154 MJ yietd.

Figure 6 . Ramges and stopping power of fast and debris ions int tungsten.

Figure 7. Ramges and stopping power of fast and debris ions int graphite.

Figure 8. Ranges and stopping power in $1 m m$ Li over $1 \mathrm{~cm}$ CFC composite wall.

Figure 9. Surface temperature rise due to direet drive target in graphite and turgstem.

Figure 10. Femperature rise due to taser, X-ray, and ionr depositions.

Figure 11. Various erosion fluxes of graphite and tungstem.

Figure 12. HEIGHFS-IFE catculation of various wall erosion mechranisms. 


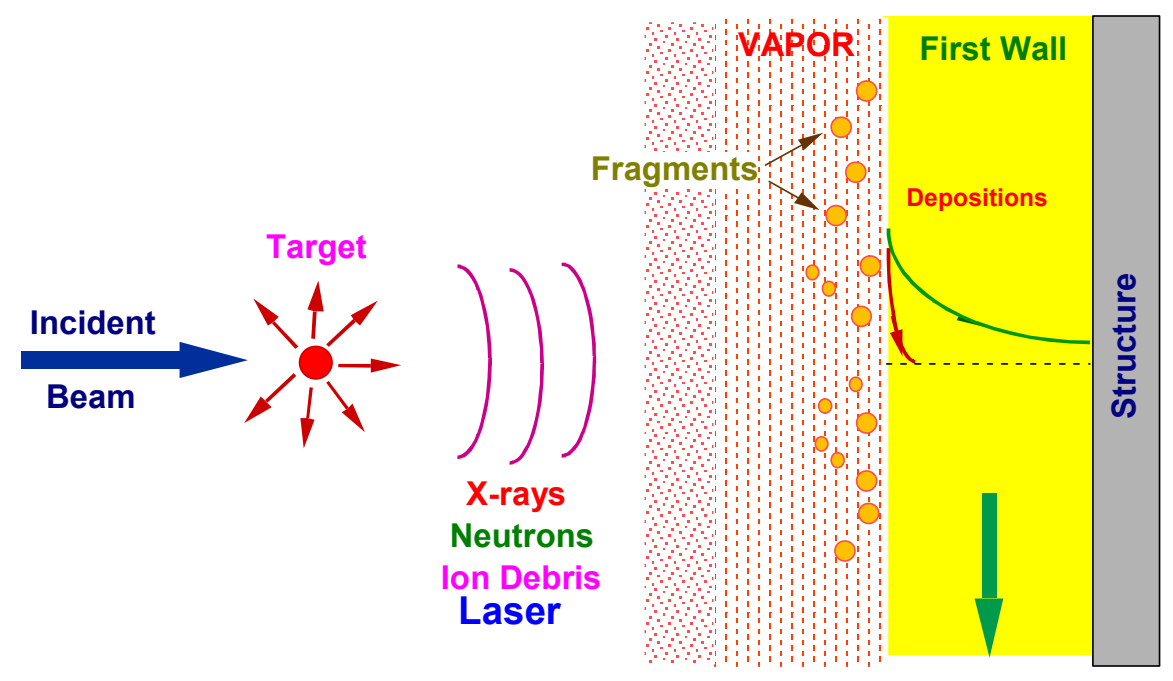

Figure 1. Schematic of energy deposition on chamber wall.

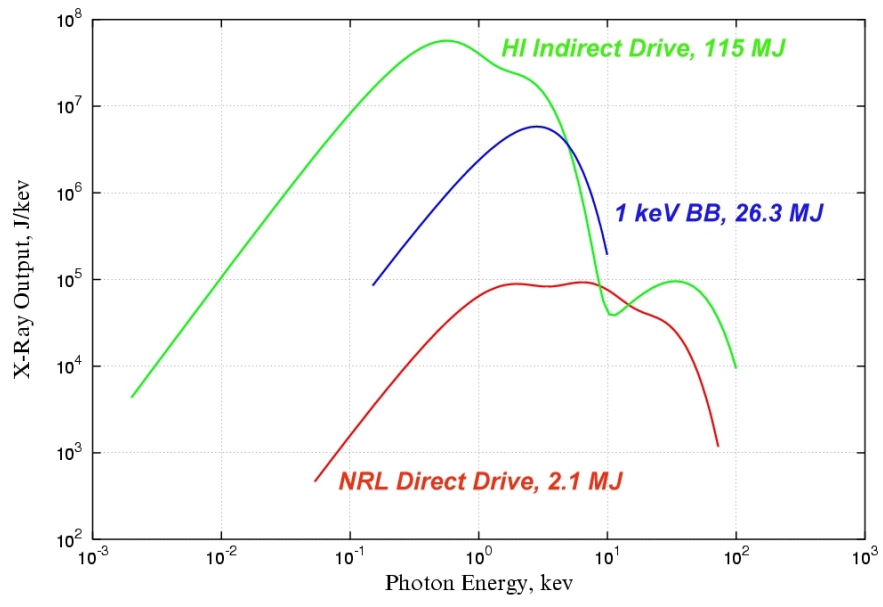

Figure 2. Photon spectra from direct and indirect target output and blackbody spectrum. 


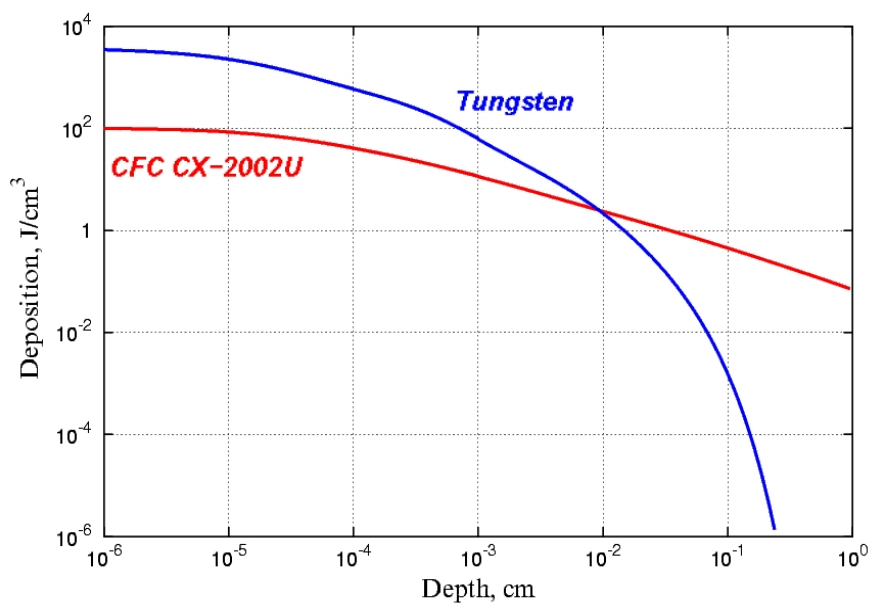

Figure 3. X-ray deposition in graphite and tungsten walls.
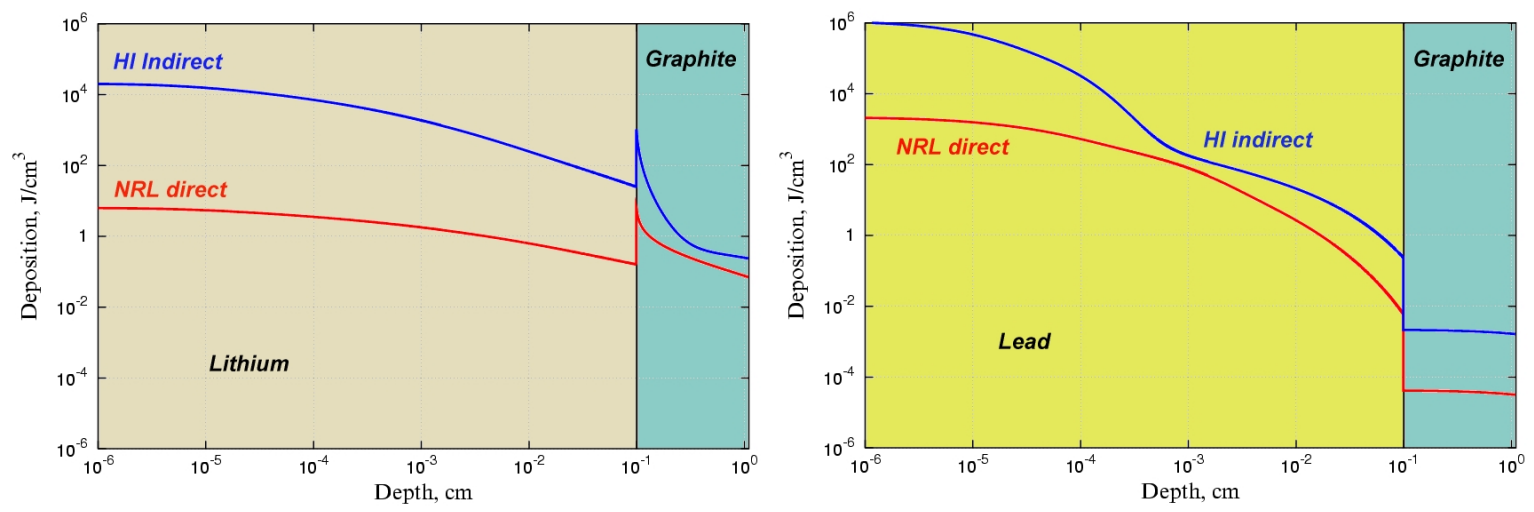

Figure 4. X-ray deposition in composite wall structure.
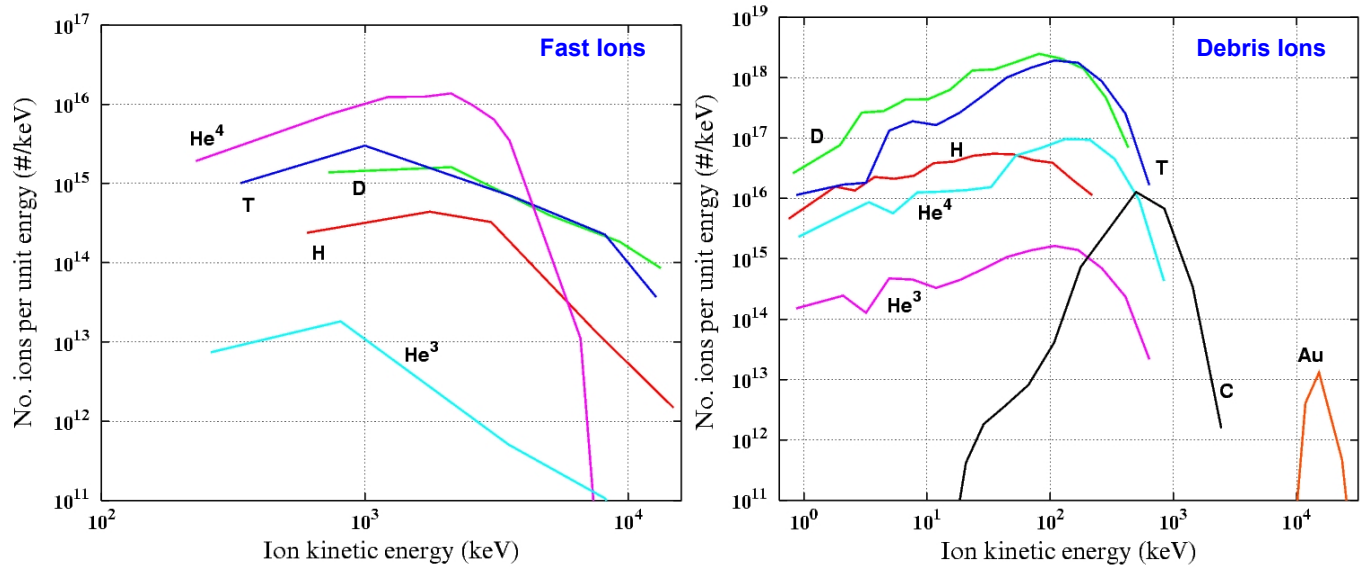

Figure 5. Particle energy distribution for NRL direct drive target of $154 M J$ yield. 

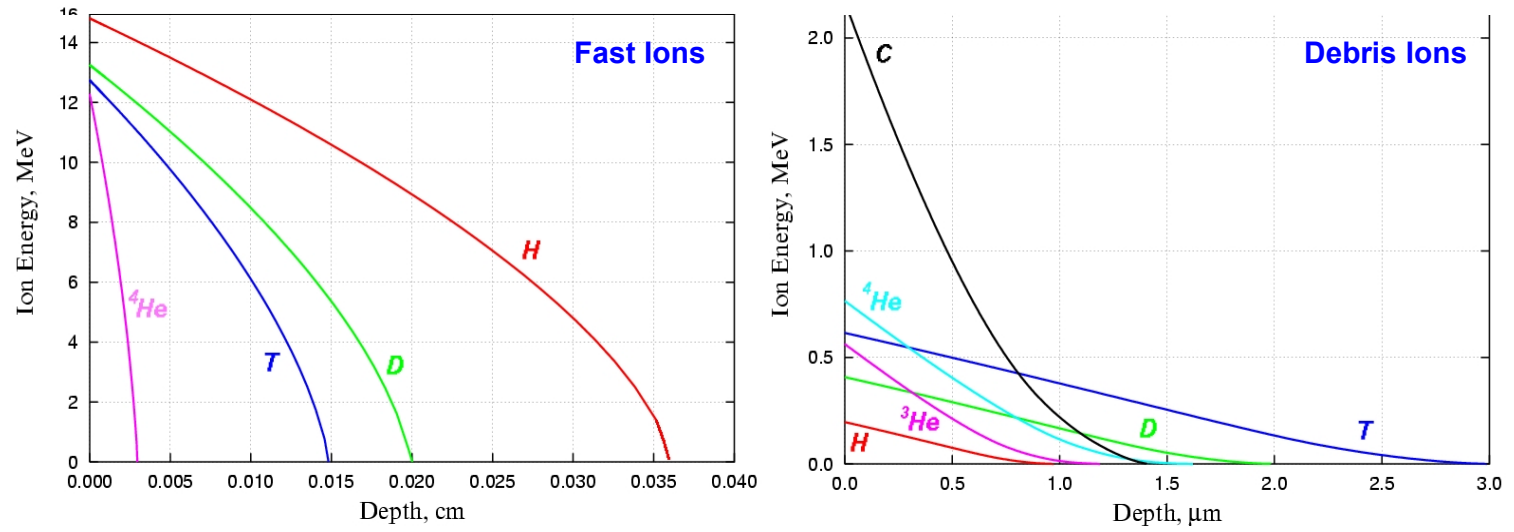

Figure 6. Ranges and stopping power of fast and debris ions in tungsten.
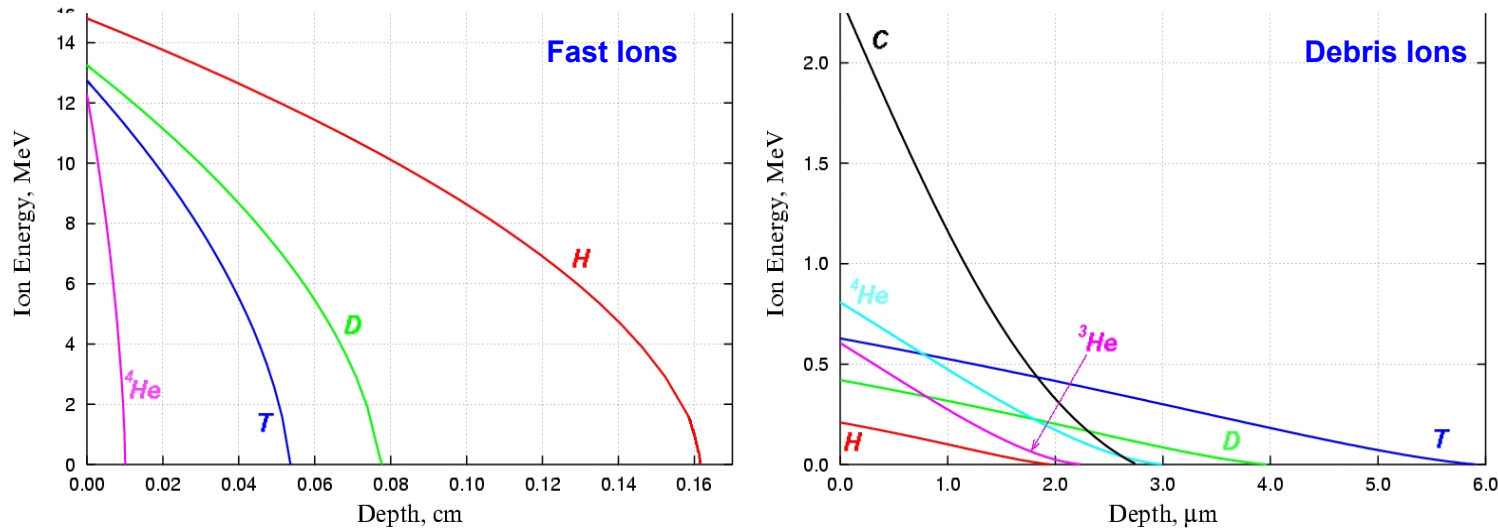

Figure 7. Ranges and stopping power of fast and debris ions in graphite.
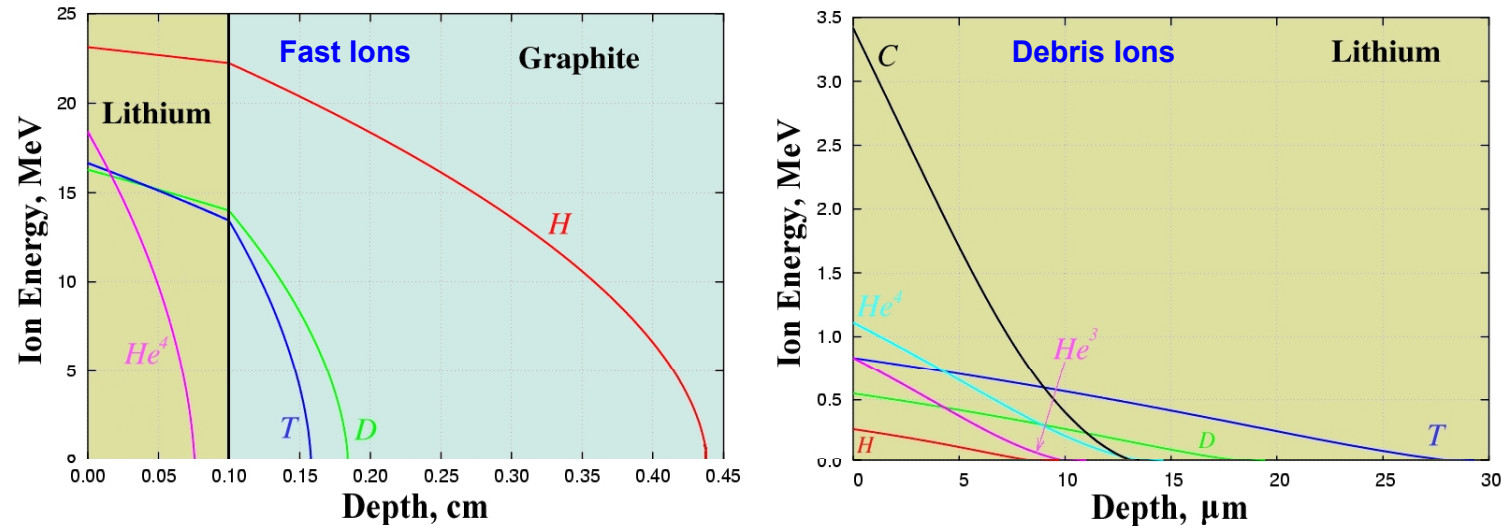

Figure 8. Ranges and stopping power in $1 \mathrm{~mm}$ Li over $1 \mathrm{~cm}$ CFC composite wall. 


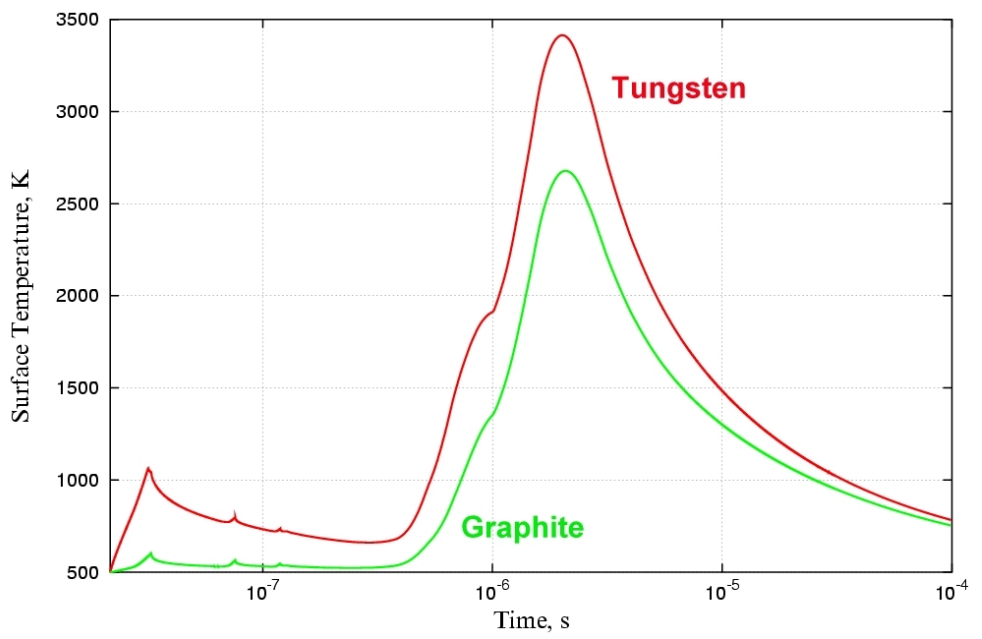

Figure 9. Surface temperature rise due to direct drive target in graphite and tungsten. 

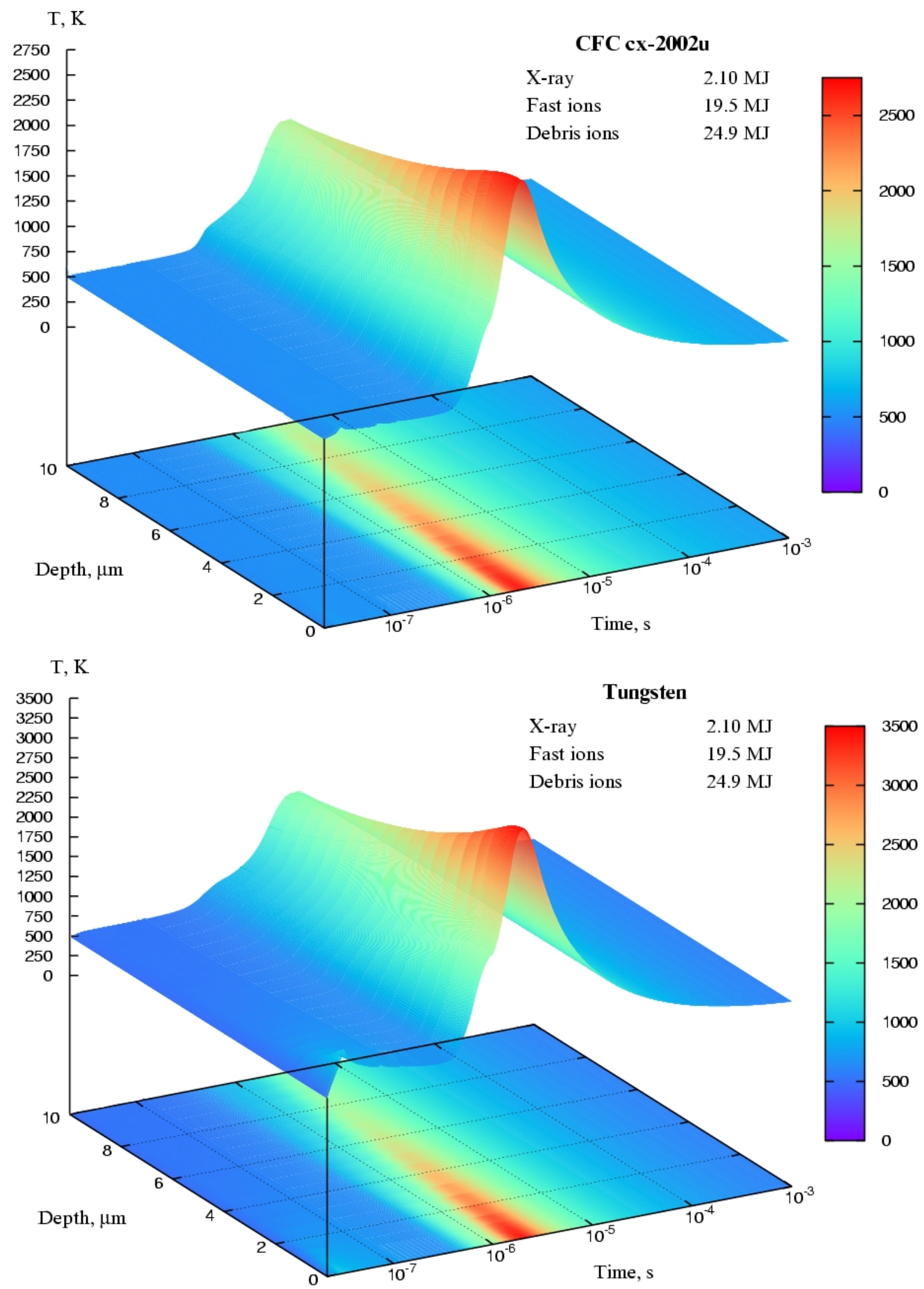

Figure 10. Temperature rise due to laser, X-ray, and ion depositions. 

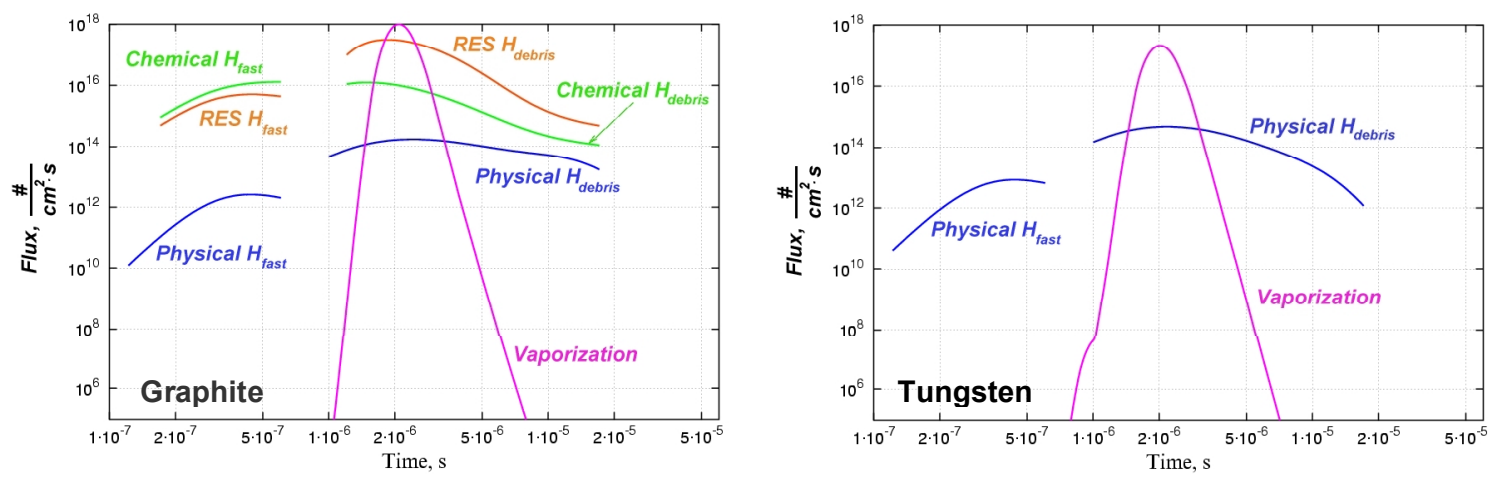

Figure 11. Various erosion fluxes of graphite and tungsten.
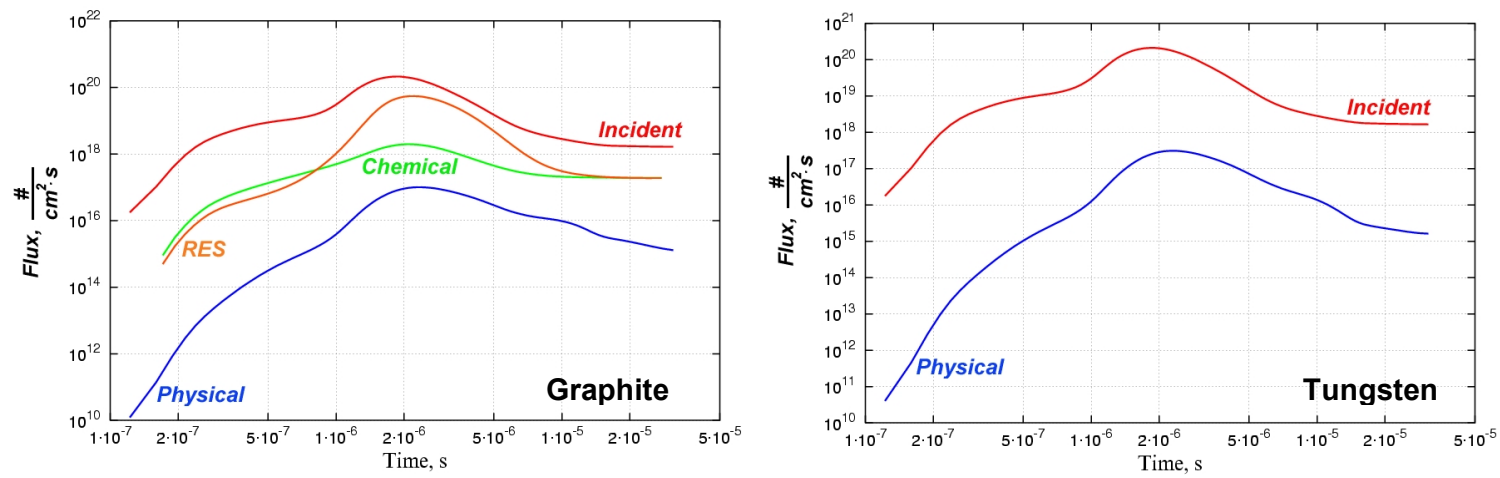

Figure 12. HEIGHTS-IFE calculation of various wall erosion mechanisms. 